\title{
Dietary Consumption of Polyphenols in University Students. Relationship with Their Health-Related Habits ${ }^{+}$
}

\author{
Maria J Rodríguez-Lagunas ${ }^{1,2, *}$, Francisco J Pérez-Cano ${ }^{1,2}$, Filipa Vicente ${ }^{3}$, Paula Pereira ${ }^{3}$ and \\ Margarida Castell 1,2,* \\ 1 Section of Physiology, Department of Biochemistry and Physiology, Faculty of Pharmacy and Food \\ Science, University of Barcelona (UB), 08028 Barcelona, Spain; franciscoperez@ub.edu \\ 2 Nutrition and Food Safety Research Institute (INSA-UB), 08921 Santa Coloma de Gramenet, Spain \\ 3 Centro de Investigação Interdisciplinar Egas Moniz, Egas Moniz Cooperativa de Ensino Superior, Quinta \\ da Granja-Campus Universitário, 2829-511 Monte da Caparica, Portugal; \\ fvicente@egasmoniz.edu.pt (F.V.); pmpereira@egasmoniz.edu.pt (P.P.) \\ * Correspondence: mjrodriguez@ub.edu (M.J.R.-L.); margaridacastell@ub.edu (M.C.) \\ + Presented at the 1st International Electronic Conference on Nutrients - Nutritional and Microbiota Effects \\ on Chronic Disease, 2-15 November 2020; Available online: https://iecn2020.sciforum.net/.
}

Published: 30 October 2020

\begin{abstract}
Polyphenols are compounds derived from plants found in foods with potential health benefits due to their antioxidant properties. The aim of this study was to establish the consumption of polyphenols in university students and the relationship between the amount of polyphenols consumed and their health-related habits. For this, 270 university students answered a validated food consumption frequency questionnaire (FFQ), a physical activity questionnaire (IPAQ), and a health and lifestyle questionnaire. From the FFQ, the daily consumption of classes and subclasses of polyphenols was calculated. Then, the population was classified in terciles according to the polyphenol's consumption, and the physical activity, smoking habits and the prevalence of allergy and obesity in each tercile was established. The consumption of polyphenols was about $1.5 \mathrm{~g} / \mathrm{day}$, being flavonoids and phenolic acids the most consumed. The main sources of polyphenol consumption were cocoa, coffee and, to a lesser extent, fruits. There were no significant differences in consumption according to sex, BMI, health status or physical activity. The smoking habit was related to a high consumption of polyphenols, mainly those that came from coffee and cocoa. Students who smoke had a higher consumption of polyphenols with respect to nonsmokers, especially those derived from coffee.
\end{abstract}

Keywords: Polyphenols; FFQ; IPAQ; smoking habit; university students

\section{Introduction}

Polyphenols are compounds from the plants's secondary metabolism that are ingested in small quantities in many edible plants [1,2]. They are classified into flavonoids, phenolic acids, lignans and stilbenes [3]. In addition, flavonoids can be classified into flavonols, flavones, isoflavones, flavanones, flavanols, and anthocyanidins.

Overall, polyphenols have a chemical structure that provide them with antioxidant activities. Due to this property, among others, they have been extensively studied in order to ascertain their possible beneficial role in the mitigation or prevention of various diseases, as evidenced by the large number of studies focused on the effect of polyphenols on health status during the last decade [1,2]. 
The available dietary assessment tools to analyze population intake include the $24 \mathrm{~h}$ dietary recalls (24HDR) and food records (3 to 7 days) [4,5]. In addition, the food frequency questionnaire (FFQ) is a valuable, cost-effective and easily applied dietary assessment tool that allow to estimate the intake of a food, food group or food component in a population and also to go in depth into its association with health parameters or disease risk [6,7].

Many different questionnaires exist to estimate polyphenol intake and they include a varied list of fruits and vegetables as well as drinks like tea, coffee cocoa and wine, in different populations [811]. However, not many of them have been applied to young people and even less aiming to establishing their relationship with health status.

Thus, we aimed to study the relationship between the amount of polyphenols consumed by university students and their health status along with their physical activity habits.

\section{Materials and Methods}

\subsection{Participants}

The study population consisted of 270 university students (199 women and 71 men) with an average age of $22.5 \pm 3.9$ years. The mean BMI was about $22 \mathrm{~kg} / \mathrm{m}^{2}$, only $7 \%$ of the participants had a BMI lower than $18.5 \mathrm{~kg} / \mathrm{m}^{2}$ and $11.5 \%$ had a BMI higher than $25 \mathrm{~kg} / \mathrm{m}^{2}$. Around $70 \%$ of the students lived with their family, $28 \%$ with flatmates, and only $2.6 \%$ lived alone. The study was conducted according to the guidelines laid down in the Declaration of Helsinki and the study protocol was approved by the Ethical Committee of the University of Barcelona (IRB 00003099) and by the Egas Moniz Ethical Commission. Written informed consent was obtained from all participants after the aims and procedure of the study were explained by members of the research team.

\subsection{Collection of Data}

Participants answered a validated food consumption frequency questionnaire (FFQ), a physical activity questionnaire (IPAQ), and a health and lifestyle questionnaire [4]. Briefly, the FFQ inquired about the frequency of consumption from 1 (never) to 12 ( 3 or more times per day of vegetables, fruits, and drinks like wine, tea or coffee. The IPAQ consisted of 7 general questions about physical activity (frequency, duration, intensity for at least $10 \mathrm{~min}$ ) in the previous 7 days. And the health status questionnaire which was based on the 'Enquesta nutricional de la població Catalana (ENCAT)' [12] included questions about smoking habits, chronic diseases and acute diseases during the previous month.

\subsection{Polyphenol Consumption Assessment}

From the data obtained in the FFQ, the amounts of polyphenol intake as well as their main sources were calculated based on the Phenol Explorer Database [13]. When several data were suitable for the same food, those obtained from chromatography study were considered. After applying Phenol Explorer Database, the consumption was assessed by multiplying the amount of polyphenol in each food by the respective consumption frequency.

After establishing the polyphenol intake of each student, the sample of 270 participants was divided into three groups of equal size and with different levels of polyphenol consumption

\subsection{Physical Activity Assessment}

From the data obtained in the IPAQ, the time dedicated to physical activity per participant as well as the metabolic equivalent of task (MET) were calculated. These data allowed the classification of students in three levels of activity: inactive ( $<600 \mathrm{METs} /$ week), moderate activity (600-1500 METs/week) and very active ( $>1500 \mathrm{METs} /$ week). 


\subsection{Statistical Analysis}

For statistical analysis, the Statistical Package for the Social Sciences (SPSS v22.0) (IBM, Chicago, IL, USA) was used. Homogeneity of variance and normality distribution were tested by the Levene's and Shapiro-Wilk tests, respectively. When data were homogeneous and had a normal behavior, Student $t$-test was used to analyze statistical differences. Otherwise, the Mann-Whitney U test was performed. Finally, the chi-square test was used to compare percentages of individuals displaying the variable in a particular tercile. Significant differences were established when $p<0.05$.

\section{Results and Discussion}

\subsection{Polyphenol Consumption: Classes and Subclasses}

The total ingestion, the class and subclass of polyphenol was established (Table 1). From all polyphenols, $49 \%$ belonged to the flavonoid family, $46 \%$ were the phenolic acids, $2 \%$ were lignans and $3 \%$ were classified as other polyphenols.

Table 1. Dairy consumption of polyphenols $(n=270)$.

\begin{tabular}{lc}
\hline \multicolumn{1}{c}{ Class } & Mean \pm SDM \\
\hline Polyphenols (mg) & $1447.3 \pm 1069.9{ }^{1}$ \\
\hline Flavonoids (mg) & $718.5 \pm 770.3$ \\
Antocyanins & $02.2 \pm 84.3$ \\
Chalcones & $0.0 \pm 0.0$ \\
Dihydrochalcones & $1.53 \pm 18.15$ \\
Dihydroflavonols & $576.4 \pm 760.3$ \\
Flavanols & $56.8 \pm 50.6$ \\
Flavanones & $6.81 \pm 6.48$ \\
Flavones & $26.55 \pm 19.18$ \\
Flavonols & $1.86 \pm 8.13$ \\
Isoflavonoids & $28.66 \pm 20.53$ \\
\hline Lignans & $0.02 \pm 0.38$ \\
\hline Non-Phenolic Metabolites & $38.5 \pm 47.6$ \\
\hline Other Polyphenols & $661.2 \pm 603.8$ \\
\hline Phenolic Acids & $0.39 \pm 0.77$ \\
\hline Stilbenes & 1 amount (mg) per day.
\end{tabular}

\subsection{Main Sources of Polyphenol and Their Main Classes}

Considering the intake of fruits, cereals, vegetables, cocoa, coffee, tea and alcoholic drinks, it has been established which food provided more polyphenols, flavonoids, flavanols and phenolic acids (Table 2). Cocoa and coffee appeared as very important sources of polyphenols, being cocoa and coffe the main source of flavonoids and phenolic acids, respectively. 
Table 2. Percentage of polyphenol and their main classes according to food group sources in the sample of study.

\begin{tabular}{ccccc}
\hline Source & Polyphenols $^{1}$ & Flavonoids & Flavanols & Phenolic Acids \\
\hline Fruits & $19.73 \pm 18.96$ & $33.05 \pm 26.25$ & $12.75 \pm 18.58$ & $12.57 \pm 16.48$ \\
Vegetables & $3.95 \pm 4.56$ & $4.24 \pm 6.13$ & $0.14 \pm 1.14$ & $5.94 \pm 9.37$ \\
Coffee & $32.73 \pm 30.17$ & $0.00 \pm 0.00$ & $0.00 \pm 0.00$ & $55.04 \pm 37.89$ \\
Cocoa & $32.35 \pm 30.74$ & $47.70 \pm 34.52$ & $65.04 \pm 33.00$ & $20.94 \pm 27.09$ \\
Cereals & $3.80 \pm 6.75$ & $0.82 \pm 3.12$ & $1.58 \pm 5.12$ & $0.15 \pm 1.16$ \\
Tea & $6.58 \pm 12.33$ & $12.52 \pm 19.61$ & $18.26 \pm 26.92$ & $4.44 \pm 10.79$ \\
Alcoholic drinks & $0.86 \pm 1.92$ & $1.67 \pm 4.92$ & $2.24 \pm 6.94$ & $0.89 \pm 2.25$ \\
\hline
\end{tabular}

1 percentage considering the entire polyphenol family category (polyphenol, flavonoids or phenolic acids).

\subsection{Distribution of Students according to Their Polyphenol Consumption}

According to their daily polyphenol consumption, students were distributed into three groups with the same number of participants: those with low consumption (lower than $839 \mathrm{mg}$, group L), those with medium consumption (between 839 and $1665 \mathrm{mg}$, group M) and those with high consumption (higher than $1665 \mathrm{mg}$, group $\mathrm{H}$ ). The mean consumption of polyphenols differed between groups and no difference in the distribution of females and males in the three categories was observed (Table 3).

Table 3. Characteristics of the participants according to the polyphenol consumption.

\begin{tabular}{cccc}
\hline Characteristics & $\begin{array}{c}\text { Low } \\
\text { Consumption }\end{array}$ & $\begin{array}{c}\text { Medium } \\
\text { Consumption }\end{array}$ & $\begin{array}{c}\text { High } \\
\text { Consumption }\end{array}$ \\
\hline Polyphenol consumption & $488.9 \pm 219.6^{\mathrm{a}}$ & $1275.0 \pm 245.5^{\mathrm{b}}$ & $2578.0 \pm 1048.4^{\mathrm{c}}$ \\
Num. females/males (\%) & $65 / 25(72 / 28 \%)$ & $72 / 18(80 / 20 \%)$ & $62 / 28(69 / 31 \%)$ \\
Physical activity (METS) & $2355.6 \pm 1965.44^{\mathrm{a}}$ & $3052.7 \pm 2684.0^{\mathrm{b}}$ & $2668.3 \pm 2886.6^{\mathrm{ab}}$ \\
${\left.\text { Body mass index (kg/m }{ }^{2}\right)}^{\text {ab }}$ & $22.1 \pm 3.0$ & $21.8 \pm 2.4$ & $21.9 \pm 2.9$ \\
Num. overweight individuals (\%) & $13(14 \%)$ & $11(12 \%)$ & $7(8 \%)$ \\
Num. non-smokers/smokers (\%) ${ }^{1}$ & $74 / 5(94 / 6 \%)$ & $69 / 5(93 / 7 \%)$ & $62 / 17(78 / 22 \%)^{*}$ \\
Mean blood pressure & $87.6 \pm 9.4$ & $85.0 \pm 8.9$ & $88.4 \pm 7.9$ \\
Num. hypertensive individuals (\%) ${ }^{2}$ & $6(11 \%)$ & $3(6 \%)$ & $7(14 \%)$ \\
Num. participants with fever (last 4 wk) (\%) & $6(7 \%)$ & $4(4 \%)$ & $12(13 \%)$ \\
Num. participants with cold (last 4 wk) (\%) & $29(32 \%)$ & $27(30 \%)$ & $28(31 \%)$ \\
Num. participants with chronic diseases (\%) & $53(59 \%)$ & $54(60 \%)$ & $49(54 \%)$ \\
Num. participants with allergy (\%) & $13(14 \%)$ & $13(14 \%)$ & $17(19 \%)$ \\
\hline
\end{tabular}

${ }^{1}$ Ex-smokers were excluded; ${ }^{2}$ Inside those that provided blood pressure values.

When considering the physical activity, METS/week were higher in the group of medium consumption than that of low consumption $(p<0.05$, Table 3$)$, while those with high polyphenol intake had an intermediate activity that did not differ from the other two groups.

The BMI, as well as the proportion of overweight individuals, did not show differences between the three groups.

The distribution of smoker and non-smoker participants, including those ex-smokers, was determined in each polyphenol consumption group. A higher percentage of smokers were found in the people with high consumption of polyphenols $(p=0.0034)$.

Participants were inquired about their blood pressure values and about $60 \%(158 / 270)$ of the students gave their values. From these, the mean blood pressure was calculated (Table 3) no differences were observed between groups according to polyphenol consumption. The percentage of hypertensive individuals (with systolic blood pressure $\geq 140 \mathrm{~mm} \mathrm{Hg}$ and or diastolic blood pressure $\geq 90 \mathrm{~mm} \mathrm{Hg}$ ) was also established and again, no differences were observed. 
The presence of fever in the last 4 weeks was also inquired to students and $4-13 \%$ students stated that they have had a higher temperature, however no differences were found with regard to their polyphenol consumption. Likewise, cold was suffered by $30-32 \%$ students in the last month and these values did not differ between the three student categories.

Finally, more than a half of the participants stated to suffer from a chronic disease, and among them, about $14-29 \%$ of the students clared to have a type of allergy. No differences were detected between groups.

\section{Conclusions}

Overall, the present study shows that cocoa and coffee are the most important sources of polyphenols in university students and that the overall polyphenol consumption do not seem to be clearly associated to a particular health status.

Funding: This research received no external funding.

Conflicts of Interest: The authors declare no conflict of interest.

\section{References}

1. Del Rio, D.; Rodriguez-Mateos, A.; Spencer, J.P.E.; Tognolini, M.; Borges, G.; Crozier, A. Dietary (poly)phenolics in human health: Structures, bioavailability, and evidence of protective effects against chronic diseases. Antioxid. Redox Signal. 2013, 18, 1818-1892.

2. Grosso, G.; Grosso; Giuseppe Effects of Polyphenol-Rich Foods on Human Health. Nutrients 2018, 10, 1089.

3. Manach, C.; Scalbert, A.; Morand, C.; Rémésy, C.; Jiménez, L. Polyphenols: Food sources and bioavailability. Am. J. Clin. Nutr. 2004, 79, 727-747.

4. Byers, T.; Lyle, B. Summary statement. In American Journal of Clinical Nutrition; American Society for Nutrition: Rockville, MD, USA, 1999; Volume 69.

5. Jacques, P.F.; Sulsky, S.I.; Sadowski, J.A.; Phillips, J.C.; Rush, D.; Willett, W.C. Comparison of micronutrient intake measured by a dietary questionnaire and biochemical indicators of micronutrient status. Am. J. Clin. Nutr. 1993, 57, 182-189.

6. Cade, J.; Thompson, R.; Burley, V.; Warm, D. Development, validation and utilisation of food-frequency questionnaires-A review. Public Health Nutr. 2002, 5, 567-587.

7. Cade, J.E.; Burley, V.J.; Warm, D.L.; Thompson, R.L.; Margetts, B.M. Food-frequency questionnaires: A review of their design, validation and utilisation. Nutr. Res. Rev. 2004, 17, 5-22.

8. Tresserra-Rimbau, A.; Medina-Remón, A.; Pérez-Jiménez, J.; Martínez-González, M.A.; Covas, M.I.; Corella, D.; Salas-Salvadó, J.; Gómez-Gracia, E.; Lapetra, J.; Arós, F.; et al. Dietary intake and major food sources of polyphenols in a Spanish population at high cardiovascular risk: The PREDIMED study. Nutr. Metab. Cardiovasc. Dis. 2013, 23, 953-959.

9. Ovaskainen, M.L.; Törrönen, R.; Koponen, J.M.; Sinkko, H.; Hellström, J.; Reinivuo, H.; Mattila, P. Dietary intake and major food sources of polyphenols in Finnish adults. J. Nutr. 2008, 138, 562-566.

10. Pérez-Jiménez, J.; Fezeu, L.; Touvier, M.; Arnault, N.; Manach, C.; Hercberg, S.; Galan, P.; Scalbert, A. Dietary intake of 337 polyphenols in French adults. Am. J. Clin. Nutr. 2011, 93, 1220-1228.

11. Zamora-Ros, R.; Knaze, V.; Rothwell, J.A.; Hémon, B.; Moskal, A.; Overvad, K.; Tjønneland, A.; Kyrø, C.; Fagherazzi, G.; Boutron-Ruault, M.C.; et al. Dietary polyphenol intake in europe: The european prospective investigation into cancer and nutrition (EPIC) study. Eur. J. Nutr. 2016, 55, 1359-1375.

12. Serra Luis, M.; Ribas Lourdes, B.; Salvador Gemma, C.; Roman Blanca, V.; Castell Conxa, A.; Cabezas Carmen, P.; Pastor Cruz, F.; Raidó Blanca, Q.; Ngo Joy, D.L.C.; García Alicia, A.; et al. Trends in the nutritional status of the Spanish population: Results from the Catalan nutrition monitoring system (19922003). Rev. Esp. Salud Publica 2007, 81, 559.

13. Rodríguez-Lagunas, M.J.; Vicente, F.; Pereira, P.; Castell, M.; Pérez-Cano, F.J. Relationship between cocoa intake and healthy status: A pilot study in university students. Molecules 2019, 24, 812.

Publisher's Note: MDPI stays neutral with regard to jurisdictional claims in published maps and institutional affiliations. 
(C) 2020 by the authors. Submitted for possible open access publication under the terms and conditions of the Creative Commons Attribution (CC BY) license (http://creativecommons.org/licenses/by/4.0/). 